\title{
A Risk Stratification Model Based on a Population Analysis for Predicting Cancer Specific Survival in Pediatric Brain Stem Glioma.
}

\author{
Kai Sun ${ }^{1}$, Xiaowei $\mathrm{Fei}^{2}$, Mingwei $\mathrm{Xu}^{3}$, Wenjin $\mathrm{Chen}^{4}$, and Ruxiang $\mathrm{Xu}^{1}$ \\ ${ }^{1}$ Sichuan Academy of Medical Sciences and Sichuan People's Hospital \\ ${ }^{2}$ Fourth Military Medical University \\ ${ }^{3}$ Third Military Medical University Daping Hospital and Research Institute of Surgery \\ ${ }^{4}$ University of Electronic Science and Technology of China Sichuan Provincial People's \\ Hospital
}

August 2, 2021

\begin{abstract}
Introduction: The aim of this study was to construct and validate a nomogram and risk stratification model for predicting cancer-specific survival (CSS) of pediatric brainstem glioma patients. Methods: Cases of pediatric brainstem glioma patients (<12 years) from 1998 to 2016 were retrieved from the Surveillance, Epidemiology, and End Results (SEER) database and demographic, clinicopathologic characteristics, treatments, and survival outcomes were analyzed. The total cohort was randomly divided into training and validation sets, followed by univariate and multivariate Cox regression analyses. A nomogram was constructed and risk stratification analysis incorporated using the selected variables from the multivariate analysis. The accuracy of the model was assessed using C-index and calibration curves. Results: A total of 806 pediatric cases with histologically confirmed diagnosis of brainstem glioma were selected and analyzed. Multivariate analysis showed that age, race, tumor size, grade and radiotherapy $(\mathrm{P}<0.05)$ were independent prognostic indicators of pediatric gliomas. For prediction of CSS, the C-index of the nomogram was 0.75 , which shows a good predictive probability. Conclusion: The nomogram developed in this study for predicting survival of pediatric patients with histologically confirmed stem gliomas is the first to incorporate risk stratification. Combining nomogram and risk stratification system is a convenient tool to aid clinicians in the identification of high-risk patients and to perform targeted adjuvant treatment.
\end{abstract}

\section{A Risk Stratification Model Based on a Population Analysis for Predicting Cancer Specific Survival in Pediatric Brain Stem Glioma}

Kai Sun ${ }^{1,2}$, Xiaowei $\mathrm{Fei}^{3}$, Mingwei $\mathrm{Xu}^{2}$, Wenjin $\mathrm{Chen}^{1}$, Ruxiang $\mathrm{Xu}^{1}$

${ }^{1}$ Department of Neurosurgery, Sichuan Academy of Medical Sciences and Sichuan Provincial People's Hospital, School of Medicine, University of Electronic Science and Technology of China, Chengdu, Sichuan, 610072, People's Republic of China;

${ }^{2}$ Department of Neurosurgery, The Third Affiliated Hospital of The Third Military Medical University, Chongqing 400042, People's Republic of China;

${ }^{3}$ Department of Neurosurgery, The First Affiliated Hospital of The Fourth Military Medical University, Xi'an, Shaan Xi, 710032, People's Republic of China;

\section{Correspondence}

Department of Neurosurgery, Sichuan Academy of Medical Sciences and Sichuan Provincial People's Hospital, School of Medicine, University of Electronic Science and Technology of China, Chengdu, Sichuan, 610072, 
People's Republic of China.

Tel +86 13391788118

Email: 1192217050@qq.com

\section{Authors' contributions}

(I) Conception and design: Kai Sun, Ruxiang Xu; (II) Administrative support: Kai Sun; (III) Provision of study materials or patients: Ruxiang Xu; Mingwei Xu; (IV) Collection and assembly of data: Xiaowei Fei, Wenjin Chen; (V) Data analysis and interpretation: Kai Sun; Xiaowei Fei, Ruxiang Xu; (VI) Manuscript writing: All authors; (VII) Final approval of manuscript: All authors.

\section{Ethics approval and consent to participate}

All analyses of human data conducted in this study were approved by the Institutional Review Board of the University of Electronic Science and Technology of China and in accordance with the ethical standards of the institutional and/or national research committee and with the 1964 Helsinki declaration and its later amendments or comparable ethical standards. All authors signed authorization forms and received permission from SEER to access and use the dataset.

\section{Consent for publication}

Not applicable

\section{Availability of data and materials}

The dataset from SEER database generated and/or analyzed during the current study are available in the SEER dataset repository (https://seer.cancer.gov/data/ ).

\section{Competing interests}

The authors declare that they have no competing interests.

\section{Funding}

This work was supported by a grant from the National Natural Science Foundation of China (Grant No.81573774), and the Military Medical Science Research Project (16CXZ001).

\section{Authors' contributions}

(I) Conception and design: Kai Sun, Ruxiang Xu; (II) Administrative support: Kai Sun; (III) Provision of study materials or patients: Ruxiang Xu; Mingwei Xu; (IV) Collection and assembly of data: Xiaowei Fei, Wenjin Chen; (V) Data analysis and interpretation: Kai Sun; Xiaowei Fei, Ruxiang Xu; (VI) Manuscript writing: All authors; (VII) Final approval of manuscript: All authors.

\section{Conflicts of interest}

The authors have no disclosures or conflicts of interest to declare.

\section{Acknowledgements}

The authors acknowledge the efforts of the Surveillance, Epidemiology, and End Results (SEER) Program tumor registries for development and maintenance of the SEER database. The authors report no conflicts of interest.

\footnotetext{
Abbreviations Table

CSS cancer-specific survival

SEER Surveillance, Epidemiology, and End Results

CNS central nervous system
} 
DPIG diffuse intrinsic pontine gliomas

\title{
A Risk Stratification Model Based on a Population Analysis for Predicting Cancer Specific Survival in Pediatric Brain Stem Glioma
}

\begin{abstract}
Background: The aim of this study was to construct and validate a nomogram and risk stratification model for predicting cancer-specific survival (CSS) of pediatric brainstem glioma patients.

Methods: Cases of pediatric brainstem glioma patients ( $<12$ years) from 1998 to 2016 were retrieved from the Surveillance, Epidemiology, and End Results (SEER) database and demographic, clinicopathologic characteristics, treatments, and survival outcomes were analyzed. The total cohort was randomly divided into training and validation sets, followed by univariate and multivariate Cox regression analyses. A nomogram was constructed and risk stratification analysis incorporated using the selected variables from the multivariate analysis. The accuracy of the model was assessed using C-index and calibration curves.
\end{abstract}

Results: A total of 806 pediatric cases with histologically confirmed diagnosis of brainstem glioma were selected and analyzed. Multivariate analysis showed that age, race, tumor size, grade and radiotherapy $(\mathrm{P}<0.05)$ were independent prognostic indicators of pediatric gliomas. For prediction of CSS, the C-index of the nomogram was 0.75 , which shows a good predictive probability.

Conclusion: The nomogram developed in this study for predicting survival of pediatric patients with histologically confirmed stem gliomas is the first to incorporate risk stratification. Combining nomogram and risk stratification system is a convenient tool to aid clinicians in the identification of high-risk patients and to perform targeted adjuvant treatment.

Keywords: Glioma, Nomogram, Prognosis, Brainstem, SEER

\section{Background}

Gliomas constitute a wide spectrum of neuroepithelial tumors arising from glia or supporting cells (astrocytes, oligodendrocytes, and ependymocytes) of the central nervous system $(\mathrm{CNS})^{1,2}$. Gliomas are responsible for nearly $24 \%$ of all primary brain and CNS neoplasms and vastly differ in histology and behavior ranging from benign ependymomal tumors to the most aggressive and lethal grade IV glioblastoma multiforme ${ }^{3}$. Specifically, brainstem gliomas are diverse groups of neoplasms that primarily affect children and include low-grade focal brainstem gliomas, as well as high-grade diffuse intrinsic pontine gliomas (DIPG) ${ }^{4,5}$. Although $80 \%$ of gliomas arise within the pons as DIPGs, the remaining low-grade gliomas are located within the midbrain, dorsal medulla, or the cervico-medullary junction. The aforementioned locations of these neoplasms pose therapeutic challenges, and hence, may negatively affect treatment outcomes.

Radiotherapy, chemotherapy, or combined treatment modalities are the standard therapeutic options for gliomas $^{6}$. Previous studies have reported failure of chemotherapy in treating DIPGs due to the lack of intratumor penetration ${ }^{7,8}$. Recently, the unraveling of the genetic landscape of DIPGs together with identification of the K27M mutation (mutation in both histones H3.1 and H3.3) has improved the understanding of the pathogenesis of gliomas and identification of novel targeted therapies ${ }^{9,10}$.

Despite a wealth of information on cancer staging, prediction of survival and treatment strategies, little is known about the determinants of cancer-specific survival in childhood brain stem glioma. The Surveillance, Epidemiology, and End Results (SEER) database of survival data from population-based cancer registries encompass $\sim 28 \%$ of the American population ${ }^{11,12}$. The aim of the current study was to characterize a comprehensive, accurate and useful prognostic model using a population-based SEER analysis to predict survival of pediatric cases of stem gliomas.

\section{Material and Methods}

\section{Ethical approval}


This is a population study with anonymized data and was therefore exempt from ethics declaration because the study was deemed not to constitute human subject research.

\section{Study Design and Patients Selection}

This population study used SEER-18 Dataset comprised 18 cancer registries across the United States. Pediatric ( $<12$ years) patients with histologically confirmed glioma diagnosed between 1998 and 2016 were included in this study. Patient data with incomplete clinical data of interest and therapy details, and poor follow up were excluded. Further, cases wherein the primary site of lesion was not brainstem were also excluded (Figure 1). Data were extracted using the SEER*Stat software (version 8.3.5) of the National Cancer Institute. Clinical characteristics retrieved in the study were prospective data on patient demographics, tumor characteristics, and survival outcomes.

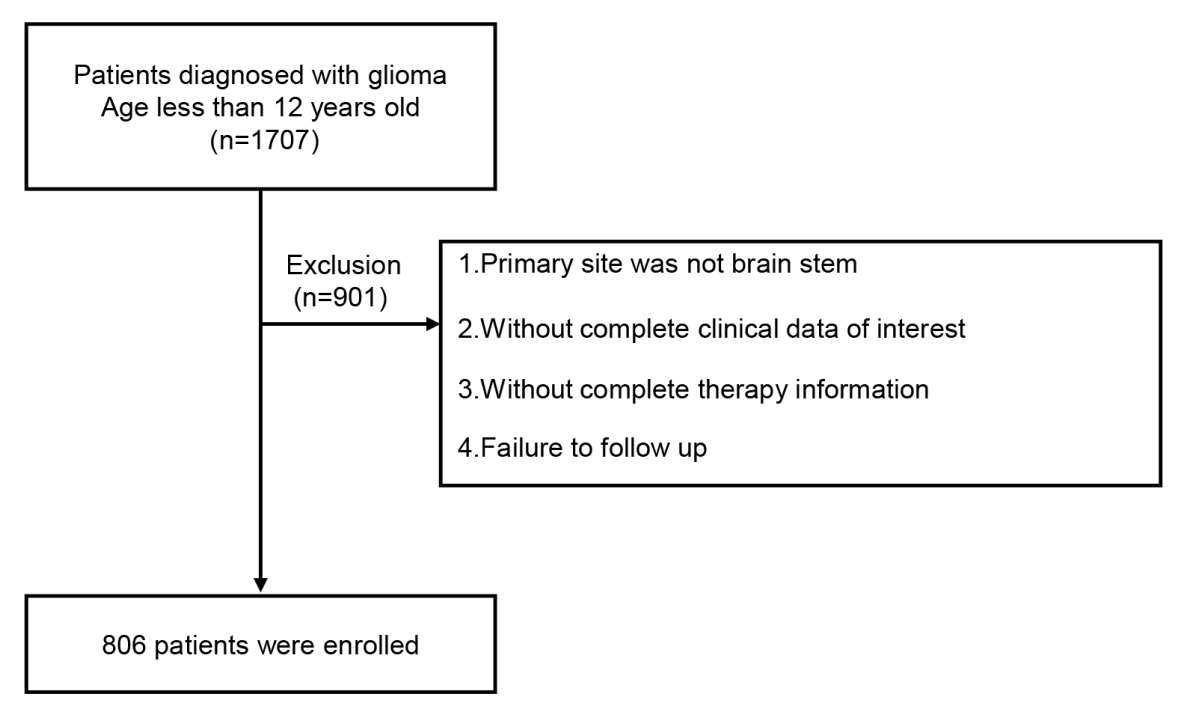

Figure 1: Patient selection of pediatric patients with brainstem gliomas within the SEER database.

\section{SEER Coding and Variable Definitions}

Demographic data included age at diagnosis, gender, and race; the latter was categorized into White/Caucasian, Black/African American and other. Tumor characteristics included histologic grade, stage, and size. Based on the study population's median value of largest tumor dimension in any direction, tumors were categorized into large $(>3 \mathrm{~cm})$, small $([?] 3 \mathrm{~cm})$, and of unknown size. Data on treatment course including surgical resection - other than biopsy, radiation therapy, and chemotherapy were retrieved from the SEER database. The era of diagnosis divided in 10-year intervals (1998-2008 and 2009-2016) was also introduced as a covariate variable.

\section{Statistical Methods}

The chi-squared test was used to compare continuous variables (rates) and categorical variables (histological grades and stage of gliomas). Kaplan-Meier plots were used for the determining the survival difference of variables. Cox regression was used for identifying risk factors of prognosis and included univariate and multivariate analyses Clinically important variables that showed significance $(\mathrm{P}<0.1)$ in the univariate analysis formed the input for multivariate analysis using the Cox risk regression model with backward elimination. Variables that showed significance $(\mathrm{P}<0.0001)$ in the multivariate analysis were selected for developing a nomogram. CSS was determined for 1, 3 and 5-years. Accuracy of the nomogram was assessed using Cindex and calibration curves generated following 1000 bootstrap resampling. A decision curve analysis is a net benefit analysis that compares the true-positive to the weighted false-positive rates across different risk thresholds that a clinician/patient might want to accept. Based on the median value of the total scores in the 
nomogram, a risk stratification model was built and comprised patients who were divided into two prognostic groups. All statistical analyses were performed using R and Empower Stats [www.empowerstats.com, XY Solutions, Inc. Boston MA]. A two-tailed $\mathrm{P}$ value $<0.05$ was considered statistically significant.

\section{Results}

\section{Patient characteristics}

The demographics, and clinicopathological characteristics of the patients are summarized in Table 1. A total of 1707 patients were screened, of which 901 were excluded (figure 1). Finally, 806 pediatric cases with histologically confirmed diagnosis of brainstem glioma were selected and analyzed. The median patient age at diagnosis was 6 years (range, 0-12). Patients were predominantly White $(\mathrm{n}=596,74 \%)$, followed by Black/African American $(\mathrm{n}=138,17.1 \%)$ and other ethnic groups $(\mathrm{n}=72,8.9 \%)$. Males $(\mathrm{n}=389,48.3 \%)$ and females $(\mathrm{n}=417,51.7 \%)$ were approximately equally distributed.

Of the 806 cases, tumor size of $87(10.8 \%)$ was considered small $(<=3 \mathrm{~cm})$ and that of $265(32.9 \%)$ were large $(>3 \mathrm{~cm})$, and size was unknown for $454(56.3 \%)$. The histological grade of most tumors was unknown $(\mathrm{n}=$ $739 ; 91.7 \%)$. Most tumors $(\mathrm{n}=720 ; 89.3 \%)$ were historic localized stage. Regarding treatment radiation therapy was the most common treatment employed for glioma $(\mathrm{n}=567,70.3 \%)$, followed by chemotherapy $(\mathrm{n}=360,44.7 \%)$; only $25(3.1 \%)$ underwent some form of surgical resection. The median follow-up duration was 11 months. Of the total, $566(70 \%)$ was randomly selected and designated as the training cohort and the remaining $240(30 \%)$ formed the internal validation cohort (Table 1).

Table 1: Patients demographics and clinicopathological characteristics of the study population

\begin{tabular}{|c|c|c|c|c|c|c|}
\hline Factors & $\begin{array}{l}\text { Entire } \\
\text { cohort } \\
(n=806)\end{array}$ & $\begin{array}{l}\text { Entire } \\
\text { cohort } \\
(n=806)\end{array}$ & $\begin{array}{l}\text { Training } \\
\text { cohort } \\
(n=566)\end{array}$ & $\begin{array}{l}\text { Training } \\
\text { cohort } \\
(n=566)\end{array}$ & $\begin{array}{l}\text { Validation } \\
\text { cohort } \\
(\mathbf{n}=\mathbf{2 4 0})\end{array}$ & $\begin{array}{l}\text { Validation } \\
\text { cohort } \\
(\mathrm{n}=240)\end{array}$ \\
\hline & $\mathbf{N}$ & $\%$ & $\mathbf{N}$ & $\%$ & $\mathbf{N}$ & $\%$ \\
\hline $\begin{array}{l}\text { Age at } \\
\text { diagnosis, } \\
\text { years }\end{array}$ & $\begin{array}{l}\text { Age at } \\
\text { diagnosis, } \\
\text { years }\end{array}$ & $\begin{array}{l}\text { Age at } \\
\text { diagnosis, } \\
\text { years }\end{array}$ & $\begin{array}{l}\text { Age at } \\
\text { diagnosis, } \\
\text { years }\end{array}$ & $\begin{array}{l}\text { Age at } \\
\text { diagnosis, } \\
\text { years }\end{array}$ & $\begin{array}{l}\text { Age at } \\
\text { diagnosis, } \\
\text { years }\end{array}$ & $\begin{array}{l}\text { Age at } \\
\text { diagnosis, } \\
\text { years }\end{array}$ \\
\hline Median & 6 & 6 & 6 & 6 & 6 & 6 \\
\hline Range & $0-12$ & $0-12$ & $0-12$ & $0-12$ & $0-12$ & $0-12$ \\
\hline \multicolumn{7}{|l|}{ Race } \\
\hline White & 596 & 74.0 & 426 & 75.3 & 170 & 70.8 \\
\hline Black & 138 & 17.1 & 101 & 17.8 & 37 & 15.4 \\
\hline Other & 72 & 8.9 & 39 & 6.9 & 33 & 13.8 \\
\hline \multicolumn{7}{|l|}{ Sex } \\
\hline Male & 389 & 48.3 & 278 & 49.1 & 111 & 46.2 \\
\hline Female & 417 & 51.7 & 288 & 50.9 & 129 & 53.8 \\
\hline \multicolumn{7}{|l|}{ YOD } \\
\hline 1998-2008 & 457 & 56.7 & 311 & 54.9 & 146 & 60.8 \\
\hline 2009-2016 & 349 & 43.3 & 255 & 45.1 & 94 & 39.2 \\
\hline \multicolumn{7}{|l|}{$\begin{array}{l}\text { Tumor size, } \\
\text { diameter, } \\
\mathrm{cm}\end{array}$} \\
\hline$[?] 3$ & 87 & 10.8 & 54 & 9.5 & 33 & 13.8 \\
\hline ¿3 & 265 & 32.9 & 197 & 34.8 & 68 & 28.3 \\
\hline Unknown & 454 & 56.3 & 315 & 55.7 & 139 & 57.9 \\
\hline \multicolumn{7}{|l|}{$\begin{array}{l}\text { Histologic } \\
\text { grade }\end{array}$} \\
\hline Well & 10 & 1.24 & 8 & 1.4 & 2 & 0.8 \\
\hline Moderately & 25 & 3.1 & 17 & 3 & 8 & 3.3 \\
\hline
\end{tabular}




\begin{tabular}{|c|c|c|c|c|c|c|}
\hline Factors & $\begin{array}{l}\text { Entire } \\
\text { cohort } \\
(n=806)\end{array}$ & $\begin{array}{l}\text { Entire } \\
\text { cohort } \\
(n=806)\end{array}$ & $\begin{array}{l}\text { Training } \\
\text { cohort } \\
(n=566)\end{array}$ & $\begin{array}{l}\text { Training } \\
\text { cohort } \\
(\mathrm{n}=566)\end{array}$ & $\begin{array}{l}\text { Validation } \\
\text { cohort } \\
(n=240) \\
\end{array}$ & $\begin{array}{l}\text { Validation } \\
\text { cohort } \\
(n=240)\end{array}$ \\
\hline Poorly & 10 & 1.24 & 7 & 1.24 & 3 & 1.3 \\
\hline Undifferentiated & 22 & 2.73 & 16 & 2.8 & 6 & 2.5 \\
\hline Unknown & 739 & 91.7 & 518 & 91.5 & 221 & 92.1 \\
\hline \multicolumn{7}{|l|}{ Stage } \\
\hline Localized & 720 & 89.3 & 506 & 89.4 & 214 & 89.2 \\
\hline Regional & 76 & 9.4 & 51 & 9.0 & 25 & 10.4 \\
\hline Distant & 10 & 1.2 & 9 & 1.6 & 1 & 0.4 \\
\hline \multicolumn{7}{|l|}{ Surgery } \\
\hline None & 781 & 96.9 & 551 & 97.3 & 230 & 95.8 \\
\hline Yes & 25 & 3.1 & 15 & 2.7 & 10 & 4.2 \\
\hline \multicolumn{7}{|l|}{ Radiotherapy } \\
\hline None & 239 & 29.6 & 169 & 29.9 & 70 & 29.2 \\
\hline Yes & 567 & 70.4 & 397 & 70.1 & 170 & 70.8 \\
\hline \multicolumn{7}{|l|}{ Chemotherapy } \\
\hline None & 446 & 55.3 & 310 & 54.8 & 136 & 56.7 \\
\hline Yes & 360 & 44.7 & 256 & 45.2 & 104 & 43.3 \\
\hline Median & 11 & 11 & 11 & 11 & 11 & 11 \\
\hline $\begin{array}{l}\text { Follow-up } \\
\text { (Months) }\end{array}$ & & & & & & \\
\hline
\end{tabular}

Abbreviations: YOD, year of diagnosis

Independent prognostic factors in the training cohort

The univariate Cox-Regression analysis showed a significant association between CSS and age at diagnosis, race, sex, tumor size, histologic grade, historic stage, chemotherapy and radiotherapy. Therefore, these variables formed the input for the multivariate Cox-Regression analysis, which revealed that age, race, tumor size, grade and radiotherapy $(\mathrm{P}<0.05)$ were independent prognostic factors (Table 2).

Table 2. Univariate and multivariate analysis for the training cohort.

\begin{tabular}{|c|c|c|c|c|}
\hline Factors & Univariate analysis & Univariate analysis & Univariate analysis & Multivariate analy \\
\hline & HR & $95 \% \mathrm{CI}$ & $\mathrm{p}^{*}$ & HR \\
\hline \multicolumn{5}{|l|}{ Age at diagnosis, years } \\
\hline 6 & 1 & & & 1 \\
\hline ¿6 & 0.6 & $0.5-0.8$ & $<0.001$ & 0.8 \\
\hline \multicolumn{5}{|l|}{ Race } \\
\hline White & 1 & & & 1 \\
\hline Black & 0.9 & $1.0-1.2$ & 0.037 & 0.8 \\
\hline Other & 1.4 & $0.9-2.0$ & 0.111 & 0.6 \\
\hline \multicolumn{5}{|l|}{ Sex } \\
\hline Male & 1 & & & 1 \\
\hline Female & 1.3 & $1.0-1.5$ & 0.028 & 1.1 \\
\hline \multicolumn{5}{|l|}{ YOD } \\
\hline 1998-2008 & 1 & & & \\
\hline $2009-2016$ & 0.9 & $0.8-1.2$ & 0.568 & \\
\hline \multicolumn{5}{|l|}{ Tumor size, diameter, $\mathrm{cm}$} \\
\hline 3 & 1 & & & 1 \\
\hline
\end{tabular}




\begin{tabular}{|c|c|c|c|c|}
\hline Factors & Univariate analysis & Univariate analysis & Univariate analysis & Multivariate analy \\
\hline ¿3 & 4.6 & $2.8-7.6$ & $<0.001$ & 1.9 \\
\hline Unknown & 2.8 & $1.7-4.5$ & $<0.001$ & 1.7 \\
\hline \multicolumn{5}{|l|}{ Histologic grade } \\
\hline Well & 1 & & & 1 \\
\hline Moderately & 0.7 & $0.2-3.0$ & 0.669 & 0.8 \\
\hline Poorly & 3.4 & $0.9-13.2$ & 0.075 & 1.5 \\
\hline Undifferentiated & 4.8 & $1.4-16.6$ & 0.013 & 2.1 \\
\hline Unknown & 2.1 & $0.7-6.7$ & 0.189 & 1.3 \\
\hline \multicolumn{5}{|l|}{ Stage } \\
\hline Localized & 1 & & & 1 \\
\hline Regional & 1.4 & $1.0-1.9$ & 0.068 & 1.2 \\
\hline Distant & 2.4 & $1.2-4.7$ & 0.009 & 1.5 \\
\hline \multicolumn{5}{|l|}{ Surgery } \\
\hline None & 1 & & & \\
\hline Yes & 0.6 & $0.3-1.2$ & 0.164 & \\
\hline \multicolumn{5}{|l|}{ Radiotherapy } \\
\hline None & 1 & & & 1 \\
\hline Yes & 5.9 & $4.3-8.2$ & $<0.001$ & 4.7 \\
\hline \multicolumn{5}{|l|}{ Chemotherapy } \\
\hline None & 1 & & & 1 \\
\hline Yes & 1.8 & $1.5-2.3$ & $<0.001$ & 1.0 \\
\hline
\end{tabular}

Abbreviations: YOD, year of diagnosis. HR, hazard ratio.

Constructing and Validating of Nomogram

Variables that were used to construct a nomogram for predicting the CSS of patients included age, race, tumor size, grade and radiotherapy $(\mathrm{P}<0.05)$ (Figure 2). Each of the independent factors were scored on a point scale axis (Table 2) and the total score calculated as the sum of each score was projected on the bottom scale and the probabilities of 1-, 3- and 5-year CSS for individual patients were thus estimated. 


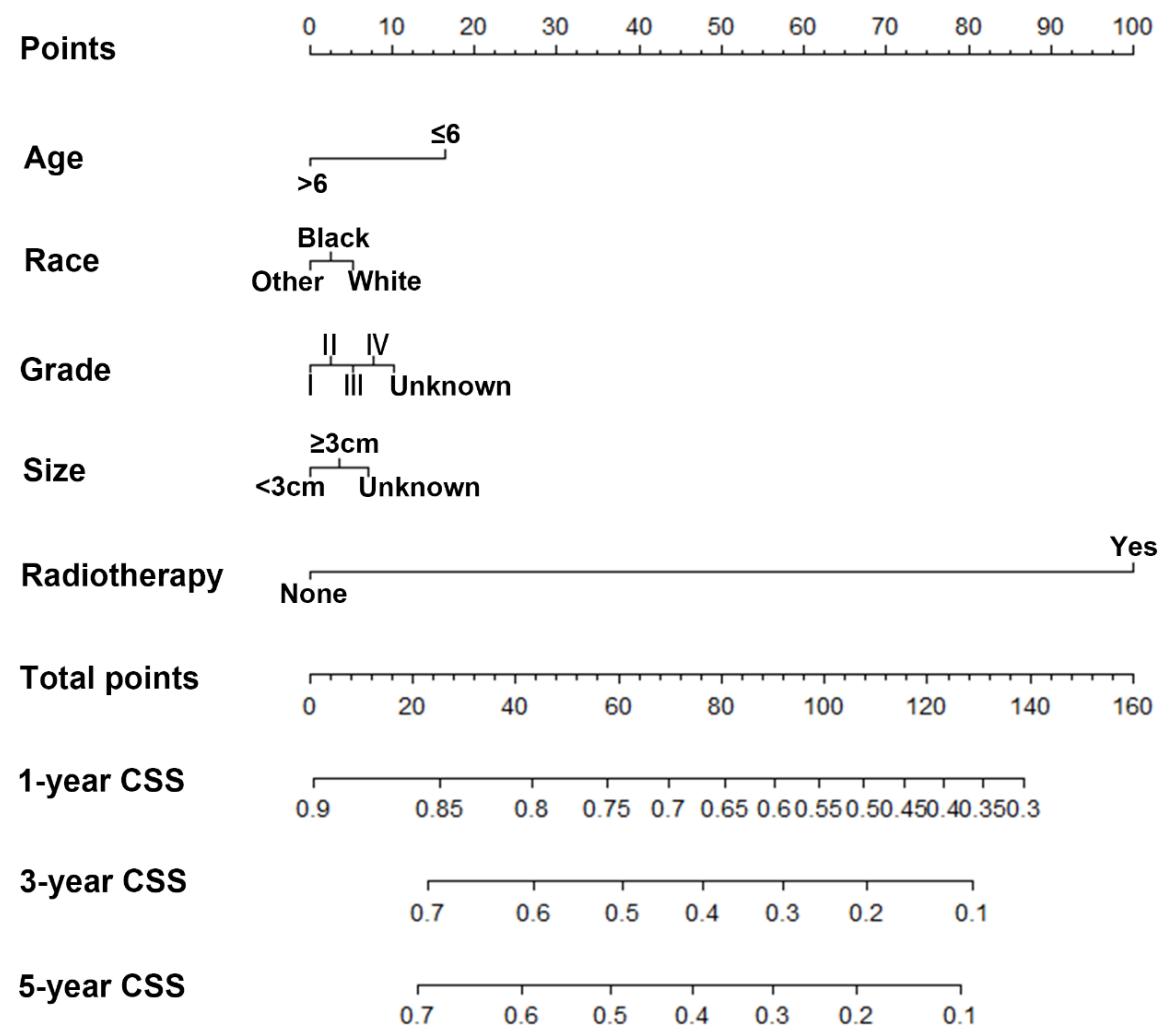

Figure 2. Nomogram predicting 1-, 3- and 5-year cancer specific survival for pediatric brainstem glioma patients.

The C-index of the nomogram thus constructed was 0.75 , which demonstrates a relatively great predictive probability. Calibration plots of the nomogram (Figure 3) demonstrated an agreement between predicted CSS and the actual observations. The decision curve analysis evaluated 1,3 and 5-year CSS of children stem glioma patients, which showed all models had a better net benefit compared to the "treat all" strategy (Figure 4).
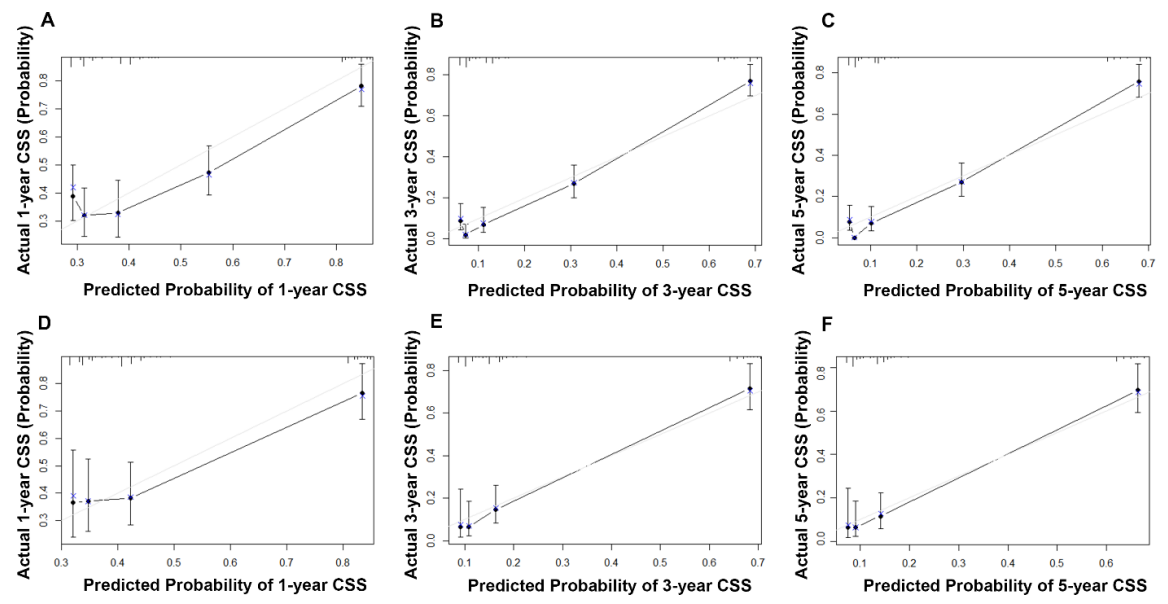

Figure 3. The calibration curves predicting 1-, 3- and 5-year cancer specific survival in the training cohort (A-C) and validation cohort (D-F). 
A

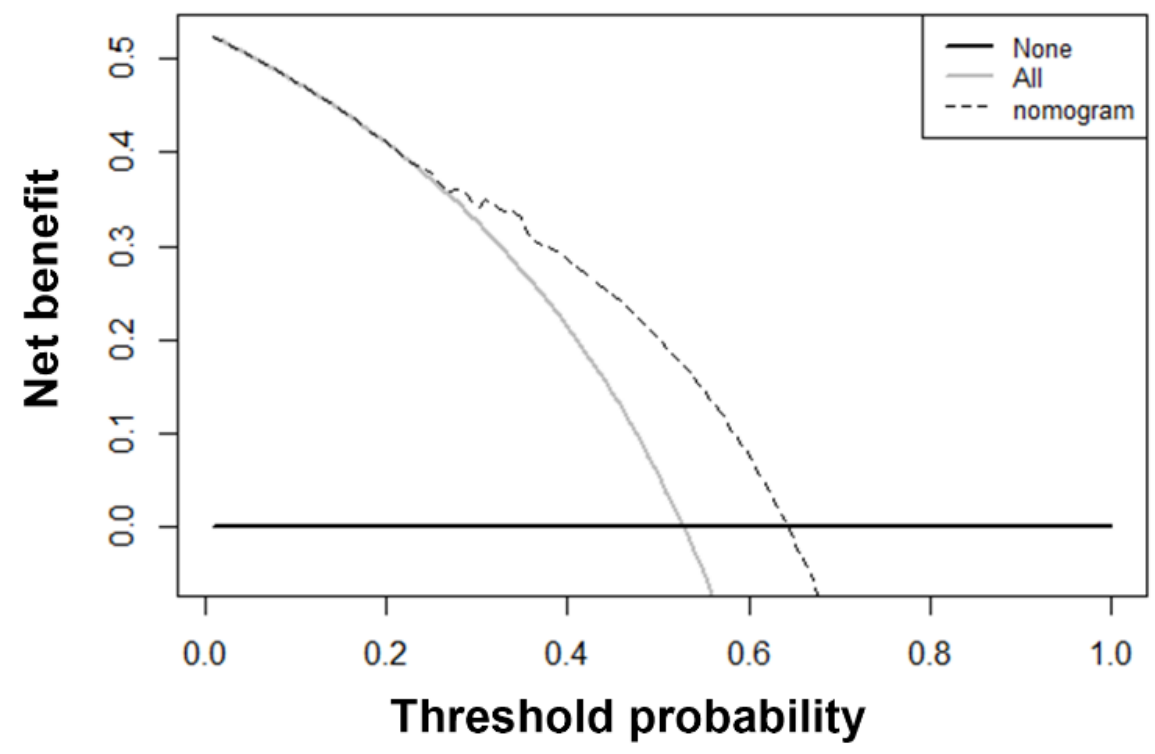

B

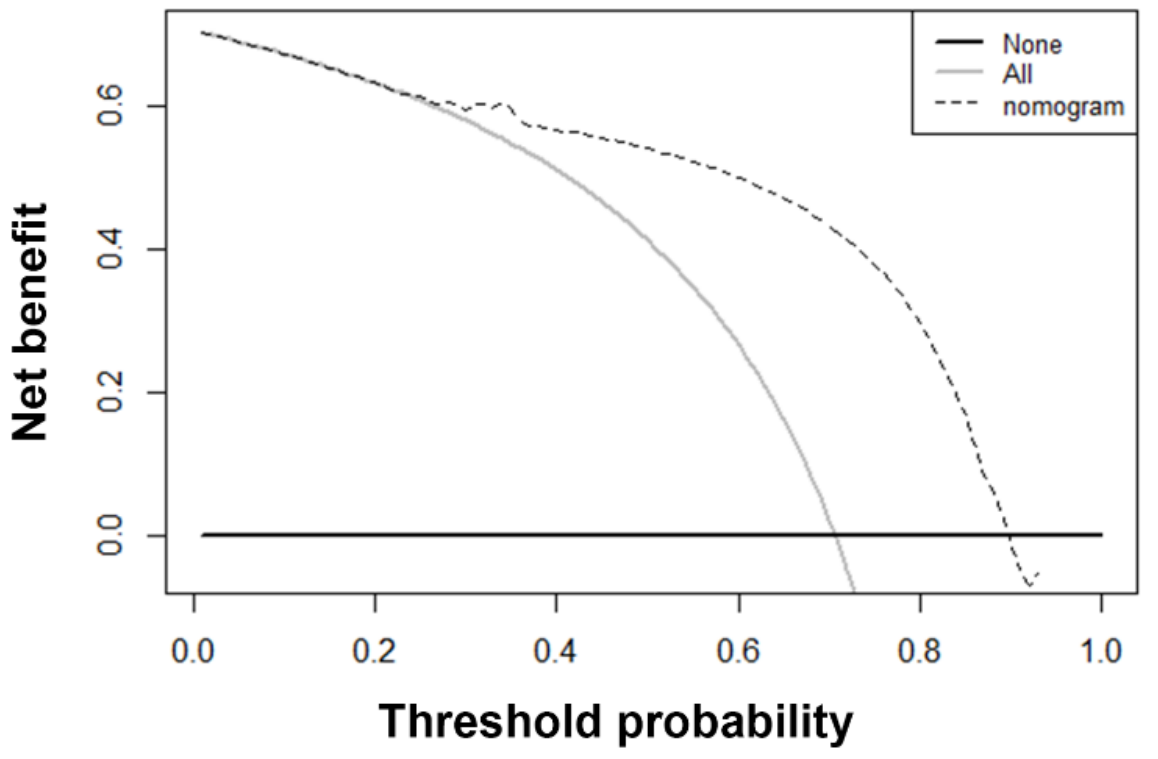

C

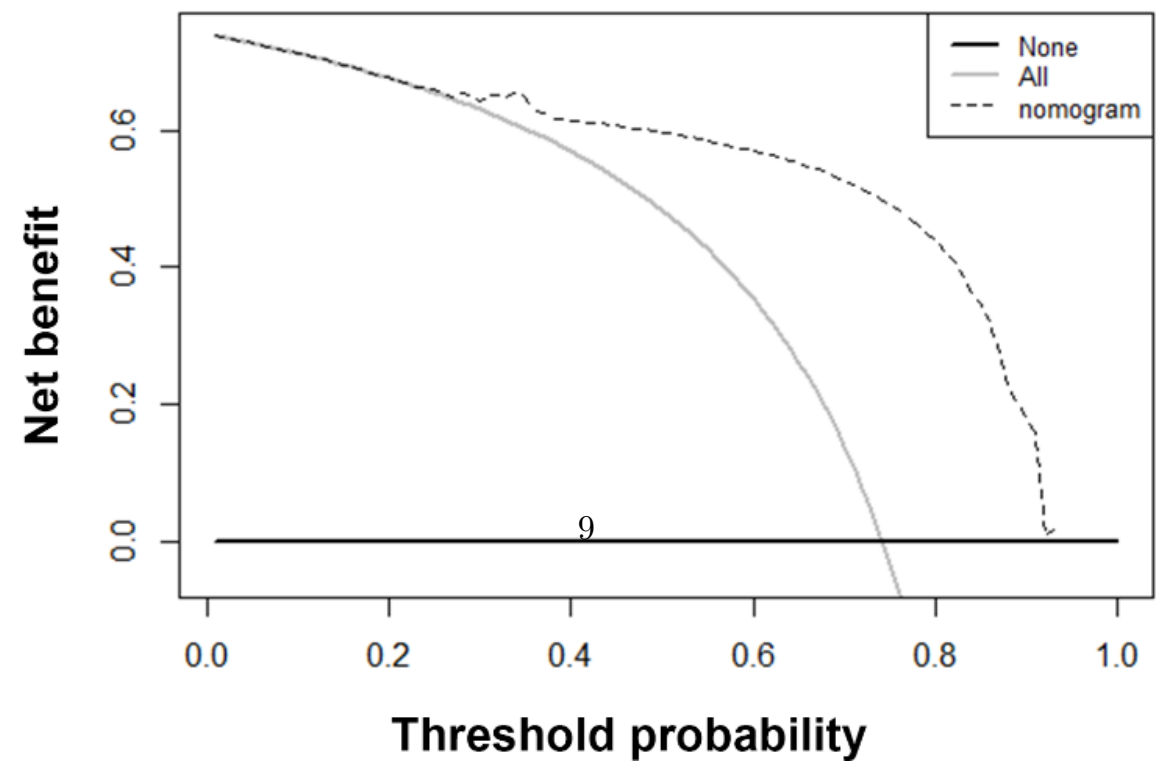


Figure 4. Decision curve analysis to show the predictive performance in 1, 3 and 5-year CSS probability (A-C). The horizontal black line represents the assumption that no patient should take the necessary measures, while the grey inverse curve represents the assumption that all patients should. The $y$-axis represents the net benefit, which was calculated by adding points associated with benefits and subtracting those associated with harms.

\section{Risk stratification system}

The total predicted score calculated from the nomogram was used in a risk stratification system for predicting patient survival. Patients were grouped into those with low (total score, < 130.78) and high (total score, [?]130.78) risk. The median survival of the entire cohort of patients with low risk, and high risk were 24 and 7 months, respectively. It showed that Kaplan-Meier survival curves predicted by the nomogram were significantly different (Figure 5F).
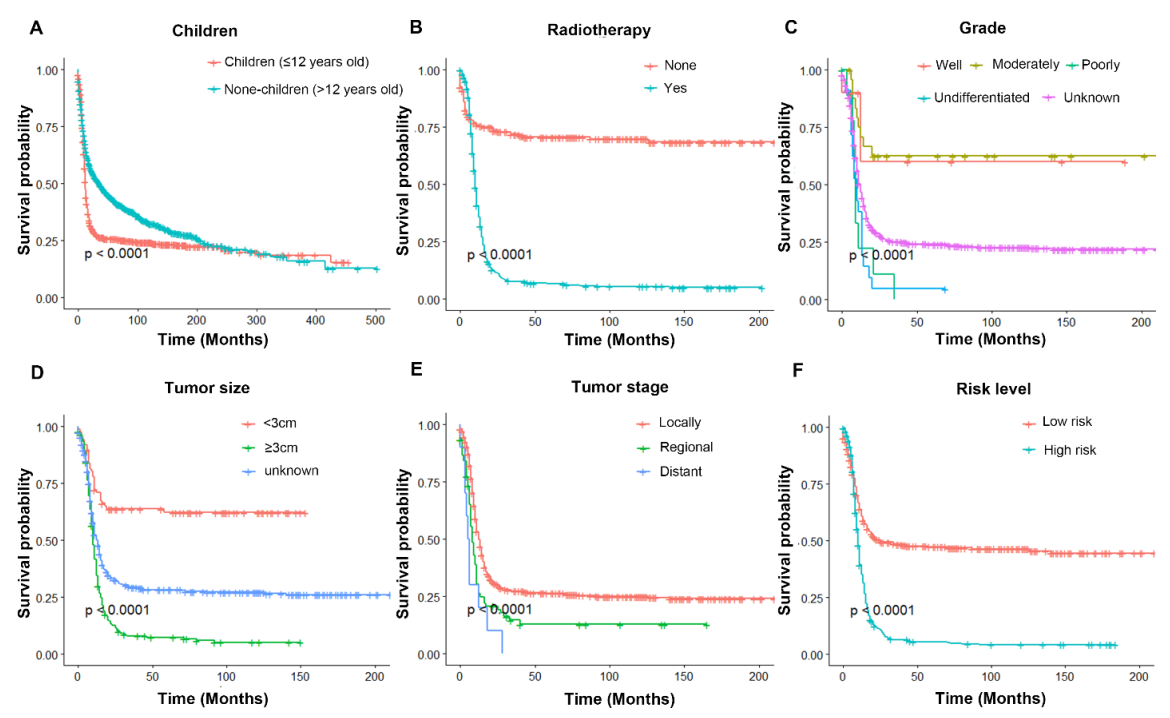

Figure 5. Kaplan-Meier curves of pediatric patients with brainstem gliomas in terms of married age (A), radiotherapy (B), historic grade (C), tumor size (D), tumor historic stage (E) and risk stratification level $(\mathrm{F})$.

\section{Discussion}

As few studies have established nomogram for predicting the survival of patients with brainstem glioma patients, the sample size involved was small, and the prognostic factors have been limited ${ }^{13}$. Thus, we developed a clinical nomogram to predict the survival based on SEER database. The SEER registry is the largest population-based database of cancer patients in the United States, covering approximately $26 \%$ of patients diagnosed with cancer in the nation. We reviewed patients' data from the latest version of the SEER as released in 2015 (covering 18 registries, 1973-2015), by using SEER*Stat version 8.3.5, and we also set a strict inclusion and exclusion criteria.

In this study, patient survival was predicted using a clinical nomogram that was based on SEER database. This was necessitated because the traditional staging classification, which is commonly used for survival predicting and clinical strategies selecting for patients with cancers cannot accurately and consistently distinguish the difference in survival among various stages. The nomogram is a comprehensive, accurate, and useful prognostic model, which has been previously used for many kinds of malignances. However, in those studies, the limitations were small sample sizes and analysis of few prognostic factors. Five independent prognostic factors: age, race, tumor size, grade and radiotherapy, identified through univariable and multivariable Cox-Regression analyses were incorporated in the clinical nomogram. Tumor histology grade 
category contributed significantly to prognosis, according to the nomogram, which is consistent, but not identical, with previous studies on survival risk factors for glioma patients, where poorly differentiated and undifferentiated status were highly associated with poor prognosis in children stem glioma patients. Most people with low-grade gliomas are treated with surgery and may receive radiotherapy thereafter. However, in this study, we found that pediatric brainstem gliomas patients had no survival benefits from radiotherapy. We also found that chemotherapy, was not an effective measure to improve outcome in cases retrieved from the SEER database ${ }^{14,15}$. There was a relatively significant effect of race on patient survival with longer median survival times in white people compared to black people.

For validation of the nomogram to guarantee that the model could be generally applied and to avoid overfitting, it was necessary to evaluate discrimination using the C-index and calibration, which was assessed by comparing the agreement between predicted and actual survival of patients ${ }^{16,17}$. Our nomogram discriminated and predicted survival more efficiently than the traditional staging system. Further, the decision curves analysis showed that our model had a better clinical net benefit across all threshold probabilities ${ }^{18,19}$. Moreover, the risk stratification system applied to two risk groups of patients could discriminate CSS in children stem glioma.

The strength of our analysis of the risk stratification system is that the nomogram was an accurate and reliable prognostic model that could aid clinicians identify high-risk patients for targeted adjuvant treatment, particularly for our highly selective cohort ${ }^{20}$. There were some certain limitations in our study. First, although we performed multivariable analysis to minimize confounder effect associated with the heterogeneities, this was a retrospective analysis, which was further compromised by the small sample size and must be accounted for when interpreting the results ${ }^{21}$. Second, the retrospective analysis may have introduced the possibility of selection bias in the study design ${ }^{22}$. Third, the SEER database lacks information on modern gene-array technology and molecular biomarkers, such as status IDH1/TERT expression ${ }^{23-26}$, which have proven to be associated with CSS in children stem glioma patients. Therefore, future prospective analysis is warranted to predict survival of pediatric cases of gliomas.

\section{Conclusion}

The novel nomogram developed in the current study for predicting survival of pediatric patients with histologically confirmed brainstem gliomas is the first to incorporate risk stratification. Therefore, combining nomogram and risk stratification system is a convenient tool to help clinicians identify high-risk patients and to perform targeted adjuvant treatment.

\section{References}

1. Chen R, Smith-Cohn M, Cohen AL, Colman H. Glioma Subclassifications and Their Clinical Significance. Neurotherapeutics : the journal of the American Society for Experimental Neuro Therapeutics.2017;14(2):284297.

2. Gusyatiner O, Hegi ME. Glioma epigenetics: From subclassification to novel treatment options. Seminars in cancer biology.2018;51:50-58.

3. Grimm SA, Chamberlain MC. Brainstem glioma: a review. Current neurology and neuroscience reports. 2013;13(5):346.

4. Mackay A, Burford A, Carvalho D, et al. Integrated Molecular Meta-Analysis of 1,000 Pediatric HighGrade and Diffuse Intrinsic Pontine Glioma. Cancer cell. 2017;32(4):520-537.e525.

5. Wu G, Diaz AK, Paugh BS, et al. The genomic landscape of diffuse intrinsic pontine glioma and pediatric non-brainstem high-grade glioma.Nature genetics. 2014;46(5):444-450.

6. Sturm D, Pfister SM, Jones DTW. Pediatric Gliomas: Current Concepts on Diagnosis, Biology, and Clinical Management. Journal of clinical oncology : official journal of the American Society of Clinical Oncology. 2017;35(21):2370-2377. 
7. Gwak HS, Park HJ. Developing chemotherapy for diffuse pontine intrinsic gliomas (DIPG). Critical reviews in oncology/hematology. 2017;120:111-119.

8. Khan DA, Laghari AA, Bari ME, Shamim MS. Treatment options for paediatric brainstem gliomas. JPMA The Journal of the Pakistan Medical Association. 2019;69(9):1400-1402.

9. Chi AS, Tarapore RS, Hall MD, et al. Pediatric and adult H3 K27M-mutant diffuse midline glioma treated with the selective DRD2 antagonist ONC201. Journal of neuro-oncology. 2019;145(1):97-105.

10. Larson JD, Kasper LH, Paugh BS, et al. Histone H3.3 K27M Accelerates Spontaneous Brainstem Glioma and Drives Restricted Changes in Bivalent Gene Expression. Cancer cell. 2019;35(1):140-155.e147.

11. Boffa DJ, Rosen JE, Mallin K, et al. Using the National Cancer Database for Outcomes Research: A Review. JAMA oncology.2017;3(12):1722-1728.

12. Doll KM, Rademaker A, Sosa JA. Practical Guide to Surgical Data Sets: Surveillance, Epidemiology, and End Results (SEER) Database.JAMA surgery. 2018;153(6):588-589.

13. Zhao YY, Wan QS, Hao Z, Zhu HX, Xing ZL, Li MH. Clinical nomogram for predicting the survival of patients with cerebral anaplastic gliomas. Medicine. 2020;99(10):e19416.

14. Donovan LE, Lassman AB. Chemotherapy Treatment and Trials in Low-Grade Gliomas. Neurosurgery clinics of North America.2019;30(1):103-109.

15. Espinoza JC, Haley K, Patel N, et al. Outcome of young children with high-grade glioma treated with irradiation-avoiding intensive chemotherapy regimens: Final report of the Head Start II and III trials.Pediatric blood \& cancer. 2016;63(10):1806-1813.

16. Balachandran VP, Gonen M, Smith JJ, DeMatteo RP. Nomograms in oncology: more than meets the eye. The Lancet Oncology.2015;16(4):e173-180.

17. Iasonos A, Schrag D, Raj GV, Panageas KS. How to build and interpret a nomogram for cancer prognosis. Journal of clinical oncology : official journal of the American Society of Clinical Oncology.2008;26(8):13641370.

18. Liang W, Zhang L, Jiang G, et al. Development and validation of a nomogram for predicting survival in patients with resected non-small-cell lung cancer. Journal of clinical oncology : official journal of the American Society of Clinical Oncology.2015;33(8):861-869.

19. Zhang G, Wu B, Wang X, Li J. A competing-risks nomogram and recursive partitioning analysis for cause-specific mortality in patients with esophageal neuroendocrine carcinoma. Diseases of the esophagus : official journal of the International Society for Diseases of the Esophagus. 2019;32(11).

20. Hoffman LM, Veldhuijzen van Zanten SEM, Colditz N, et al. Clinical, Radiologic, Pathologic, and Molecular Characteristics of Long-Term Survivors of Diffuse Intrinsic Pontine Glioma (DIPG): A Collaborative Report From the International and European Society for Pediatric Oncology DIPG Registries. Journal of clinical oncology : official journal of the American Society of Clinical Oncology.2018;36(19):1963-1972.

21. Zhao YY, Chen SH, Hao Z, Zhu HX, Xing ZL, Li MH. A Nomogram for Predicting Individual Prognosis of Patients with Low-Grade Glioma. World neurosurgery. 2019;130:e605-e612.

22. Yang $\mathrm{T}$, Mao $\mathrm{P}$, Chen X, et al. Inflammatory biomarkers in prognostic analysis for patients with glioma and the establishment of a nomogram. Oncology letters. 2019;17(2):2516-2522.

23. Chen H, Judkins J, Thomas C, et al. Mutant IDH1 and seizures in patients with glioma. Neurology. 2017;88(19):1805-1813.

24. Picca A, Berzero G, Di Stefano AL, Sanson M. The clinical use of IDH1 and IDH2 mutations in gliomas. Expert review of molecular diagnostics. 2018;18(12):1041-1051. 
25. Zhou L, Wang Z, Hu C, et al. Integrated Metabolomics and Lipidomics Analyses Reveal Metabolic Reprogramming in Human Glioma with IDH1 Mutation. Journal of proteome research. 2019;18(3):960-969.

26. Eckel-Passow JE, Lachance DH, Molinaro AM, et al. Glioma Groups Based on 1p/19q, IDH, and TERT Promoter Mutations in Tumors. The New England journal of medicine. 2015;372(26):2499-2508.

Figure Legend

Figure 1: Patient selection of pediatric patients with brainstem gliomas within the SEER database.

Figure 2. Nomogram predicting 1-, 3- and 5-year cancer specific survival for pediatric brainstem glioma patients.

Figure 3. The calibration curves predicting 1-, 3- and 5-year cancer specific survival in the training cohort (A-C) and validation cohort (D-F).

Figure 4. Decision curve analysis to show the predictive performance in 1, 3 and 5-year CSS probability (A-C). The horizontal black line represents the assumption that no patient should take the necessary measures, while the grey inverse curve represents the assumption that all patients should. The y-axis represents the net benefit, which was calculated by adding points associated with benefits and subtracting those associated with harms.

Figure 5. Kaplan-Meier curves of pediatric patients with brainstem gliomas in terms of married age (A), radiotherapy (B), historic grade (C), tumor size (D), tumor historic stage (E) and risk stratification level $(\mathrm{F})$. 